\title{
Ideas about difficult situations and other people among students with social frustrations
}

\author{
Valeriya Alperovich ${ }^{1}$ and Anna Korochentseva ${ }^{2 *}$ \\ ${ }^{1}$ Academy of Psychology and of Pedagogics, Southern Federal University, 116, Dneprovskiy lane, \\ Rostov-on-Don, 344065, Russia \\ ${ }^{2}$ Don State Technical University, 1, Gagarin sq., Rostov-on-Don, 344003, Russia
}

\begin{abstract}
The article describes the problem of psychosocial personal characteristics as predictors of the coping behavior. Over the past few decades, the world has changed beyond recognition, the current generation of students has changed, and therefore the strategies that they use to cope with the difficulties that have appeared in their lives have also changed. The ways of their adaptation to modern reality have changed in comparison with the older generation. In this regard, the study of various predictors of coping behavior, which today prevail in the modern student environment, becomes relevant. The authors used content-analysis of metaphors, tests and methods of mathematical statistics (quartiles, regression analysis, Kruskal-Wallis H-test) and discovered the dependence between social frustration, metaphors of difficult life situations and copingstrategies. So, a high level of social frustration is connected with low rational and adaptive coping strategies in difficult life situations. Various coping strategies are associated with different metaphors of difficult life situations and of their participants: "friends" and "aliens". The metaphors reflect personal perception of these situations based on scales "short distance-long distance", "rational value-emotional value" and on parameters "undertaking the responsibility - placing the responsibility with others", "stereotypical or differentiated image of the situation".
\end{abstract}

\section{Introduction}

The studies of coping behavior (coping) (R. Lazarus, S. Folkman) in adult personalities are relevant in the contemporary social situation in various countries of the world going through economic transformations and crises, aggravation of social instability, and interethnic conflicts fraught with people's losing their jobs and being forced to migrate. Russian psychologists (T.L. Kryukova, M.V. Saporovskaya) [1, 2, 3] characterize coping behavior as a focused, constructive and conscious way of responding. Both foreign (J. de la Fuente; M. Nicolas; C. Vizoso; P. Zepp et al.) [4, 5, 6, 7] and Russian psychology (T.V. Gushchina, E.A. Doryeva, T.L. Kryukova, M.V. Saporovskaya et al.) [1, 2, 3, 8] study coping behavior, various kinds of coping strategies and their interrelations with social and

\footnotetext{
* Corresponding author: anna-kor@bk.ru
} 
psychological phenomena and properties of personality acting as predictors of the former: self-efficiency, self-esteem, self-acceptance, locus of control, anxiety level, age, gender, belonging to a certain group (ethnic, social and cultural), the experience gained in the family and social institutions, social role, mass media, religious denomination, independent or dependent "I", individual values, etc.

The social frustration extent level, i.e. that of displeasure of one's system of relationships with others and the society in general, becomes one of the social and psychological properties of the subject (O.A. Bazhukova, A.L. Bubnov, N.A. Chernova, T.D. Dubovitskaya, I.A. Krasilnikov et al.). According to various psychologists, an individual experiences the condition of frustration in situations the individual perceives as unfamiliar, new, difficult, as obstacles to carrying out an activity emerge $[9,10,11]$. In Russian psychology (E.V. Bityutskaya, E.V. Gagai, N.V. Kalinina, I.V. Kochkareva, I.V. Kochkareva, G. Yu. Martiyanova, O.Yu. Matsukevich et al.), the interrelations between one's perception of difficult life situations and one's self-attitude, self-esteem, "I" concept etc. are studied $[14,15,16,17,18,19,20]$.

Studies by Russian scientists have shown that the emotional sphere of the personality also affects the attitude towards one's "physical self". The experiences that a person encounters change his attitude towards his appearance. It behaves differently, and looks differently, depending on the coping strategy chosen [13]. Satisfaction or dissatisfaction with one's external appearance, external image, broadcast to others, in turn, is an emotionally saturated component of the value-semantic sphere of the subject and also affects the experience of the individual, relationship with social reality, affects the assessment of his life, himself, his prospects (E.V. Belugina; V.A.Labunskaya). Thus, the choice of coping behavior strategy is manifested in both internal and external personality changes. Dissatisfaction with oneself, one's behavior, and life situation, as a whole, may be associated with the system of a person's attitudes towards oneself, self-perception and general dissatisfaction with oneself in various spheres of a person's life, personal, professional, etc.

The authors have found some controversies in the research of coping strategies. Their interrelations with social and psychological properties of personality are analyzed but their interrelations with the extent level of social frustration of personality are studied little if any. The phenomenon of a "difficult life situation" as an object of coping is discussed but the interrelations of one's coping strategies and ideas about difficult situations that influence one's coping behavior are understudied.

\section{Materials and Methods}

The problem of this empirical research was social and psychological properties of personality as predictors of the personality's strategies of coping behavior (coping strategies). The research objectives consisted in identifying the influence of social frustration and metaphoric images of difficult life situations on the subjects' coping strategies as well as in identifying the distinctions of coping strategies of subjects who differ in the social frustration extent level and metaphors of difficult life situations. The subject of the research comprises coping strategies, the social frustration extent level, and metaphors of difficult life situations in participants of the research. The following hypotheses of the research have been worded: 1. Social frustration and metaphoric images of difficult life situations can influence the subjects' coping strategies; 2. Coping strategies can differ in people having different levels of extent of social frustration; 3. Coping strategies can differ in people having different metaphors for difficult life situations.

The following methods of research were used: content analysis of metaphors, testing, mathematical statistics methods (quartiles, regression analysis, Kruskal-Wallis H-test). 
The following research techniques were employed: 1. the author's technique "Metaphors of a difficult life situation" (V.D. Alperovich, 2017) developed on the basis of the "Incomplete sentences" method; 2. L.I. Vasserman's technique "Express diagnostics of social frustration level"; 3. Ways of Coping Questionnaire by R. Lazarus and S. Folkman (as adapted by T.L. Kryukova, E.V. Kuftyak and M.S. Zamyshlyaeva, 2004). The research engaged 101 respondents in their emerging adulthood - aged 18-25 - as the empirical object (students of Southern Federal University and employees of various enterprises of Rostov-on-Don). The validity of the results obtained was ensured by using in the research the mathematical statistics methods and the standard software package for statistical data processing IBM SPSS Statistics 20.0.

\section{Results}

At the first stage of the empirical research, the extent level of social frustration was identified in the respondents and their metaphors for a difficult life situation have been found out. The kinds of the respondents' difficult life situation metaphors and the participants of the latter ("friends" and "aliens") are identified using the classifier of metaphors as follows (V. D. Alperovich, 2016): 1) anthropomorphic metaphors for subjects of difficult life situations (role metaphors) (for example, "relatives", "family", "close ones") ("black mafia", "adversaries", "sectarian", "terrorist"); 2) attribute metaphors for both subjects and situations (e.g. "warm ones"-"cold ones"); 3) metaphors of similarity and distinction for subjects of difficult life situations (for example, "kindred spirits", "similar to me", "of the same dough", "ones who speak another language", "extraterrestrials"); 4) abstract metaphors of difficult life situations (e.g., "the good", "happiness", "coziness", "light", "relaxation", "warmth", "ease") ("the evil", "pain", "emptiness", "obstacle"); 5) precedent name metaphors for subjects of difficult life situations (including positive and negative fairytale and real characters) (for example, "Winnie the Pooh bear", "hero knight") ("the Trojan horse", "vampire", "werewolf"); 6) zoomorphic metaphors for subjects and situations (pets, small ones) (e.g., "fluffy kind little animals", "devoted dogs", "kind cats") (beasts of prey) (such as "unpredictable tigers", "wicked dogs", "snakes", "sharks"); 7) artifact metaphors for subjects and situations useful objects associated with positive emotions) (for example, "a warm plaid", "colorful crayons", "a life ring") (harmful objects or ones associated with negative emotions) (such as "needles", "sandpaper", "manure"); 8) naturomorphic metaphors for subjects and situations (natural objects and phenomena, associated with positive and negative emotions) ("rays of the Sun", "fresh herbs", "home flowers" etc.) ("stormy sky", "ice-cold wind", "thick clouds"); 9) organism metaphors reflecting the perception of subjects (e.g. "a reliable strong shoulder") and situations ("health-illness"); 10) "magic" metaphors for subjects and situations (for example, "an angel", "a caring wizard", "wizardry"); 11) metaphors of quality and significance for subjects and situations (e.g. "a pattern of communication"); 12) social metaphors (such as political ones ("government", "elections"), economic ones ("inflation", "crisis"), and transport ones ("like by plane", "like by car"). Next, the research participants' strategies of coping behavior were identified.

The second stage of the empirical research involved calculating quartiles with the values of the respondents' social frustration extent level. As a result, the sampling was subdivided into 4 groups. Group 1 of the respondents features the lowest extent level of social frustration, with the parameter being greater than or equal to 0 but smaller than or equal to 0.975 . Group 2 of the respondents is distinguished by a medium social frustration extent level which is close to the low one, the parameter being greater than 0.975 but smaller than or equal to 1.35. Group 3 united the respondents having a medium extent level of social frustration, with the parameter greater than 1.35 but smaller than or equal to 1.775 . In group 
4 having a higher social frustration level, the parameter is greater than 1.775. By means of Kruskal-Wallis H-test, the comparative analysis of coping strategies and metaphors for a difficult life situation in group 4 of the respondents was conducted. The results are given in Table 1.

Table 1. Comparative analysis of particularities of coping strategies and difficult life situation metaphors in the respondents that differ in the social frustration extent level.

\begin{tabular}{|l|c|c|c|c|c|}
\hline $\begin{array}{c}\text { Names of } \\
\text { coping } \\
\text { strategies / } \\
\text { kinds of } \\
\text { difficult } \\
\text { situation } \\
\text { metaphors }\end{array}$ & $\begin{array}{c}\text { Low social } \\
\text { frustration } \\
\text { level } \\
\text { respondents }\end{array}$ & $\begin{array}{c}\text { Medium social } \\
\text { frustration } \\
\text { level } \\
\text { respondents }\end{array}$ & $\begin{array}{c}\text { Medium social } \\
\text { frustration } \\
\text { level } \\
\text { respondents }\end{array}$ & $\begin{array}{c}\text { Highest social } \\
\text { frustration } \\
\text { level } \\
\text { respondents }\end{array}$ & $\begin{array}{c}\text { Asymp. } \\
\text { Sig. }\end{array}$ \\
\hline $\begin{array}{l}\text { Coping } \\
\text { strategy } \\
\text { "Planning" }\end{array}$ & 62.86 & 53.52 & 55.54 & 32.14 & 0.002 \\
\hline $\begin{array}{l}\text { Neutral and } \\
\text { ambivalent } \\
\text { artifact } \\
\text { metaphors }\end{array}$ & 46.18 & 55.05 & 60.93 & 42.14 & 0.041 \\
\hline $\begin{array}{l}\text { Metaphors } \\
\text { of quality }\end{array}$ & 45.90 & 56.00 & 53.80 & 47.92 & 0.053 \\
\hline
\end{tabular}

According to the data obtained, the respondents featuring a low social frustration extent level tend to opt for coping strategy "Planning" the most frequently, and, conversely, the respondents having the highest extent level of this parameter choose this coping strategy the least. The respondents with a higher social frustration extent level are less prone to describe a difficult situation using neutral and ambivalent artifact metaphors than the respondents having the low and medium extent levels of this parameter. Thus, they are less prone to evaluating it in a differentiated manner, to singling out various sides and aspects, to perceiving it as a controllable one than the respondents in that social frustration is less pronounced. The respondents having a low extent level of social frustration tend to use metaphors of quality and significance less than other respondents. Therefore, they hyperbolize difficult situations the least of all. At the third stage of the empirical research, regression analysis of the parameters was performed in order to identify the impact of the social frustration extent level and difficult situation metaphors on the coping strategies. In the regression model, $34.9 \%$ of changes of the values of the "confrontational coping" strategy are due to the influence of the extent of social frustration and metaphors of difficult situations, the significance level being high (0.002) (see Table 2).

Table 2. Results of regression analysis of the impact of social frustration and metaphors of difficult situations on coping strategy "confrontational coping".

\begin{tabular}{|c|c|c|c|c|}
\hline $\mathbf{R}$ & R Square & Adjusted R Square & F & Sig. \\
\hline 0.349 & 0.122 & 0.104 & 6.737 & 0.002 \\
\hline
\end{tabular}

The respondents' choosing this coping strategy is mostly influenced by negative artifact metaphors and positive precedent name metaphors. This means the respondents voting for confrontational coping tend to perceive difficult situations as controllable ones more. They may be prone to "polar" estimations of difficult situations and communication partners involved in them: to distancing, "objectifying" its negative aspects and subjects perceived 
by them as "aliens", "adversaries" while enhancing the attribution of positive qualities to subjects they perceive as "friends" and "allies". In the regression model, $46.9 \%$ of changes of the values of the "planning" coping strategy are due to the influence of the extent of social frustration and metaphors of difficult situations, the significance level being high (0.000) (see Table 3).

Table 3. Results of regression analysis of the impact of social frustration and metaphors of difficult situations on coping strategy "Planning".

\begin{tabular}{|c|c|c|c|c|}
\hline R & R Square & Adjusted R Square & F & Sig. \\
\hline 0.469 & 0.220 & 0.204 & 13.704 & 0.000 \\
\hline
\end{tabular}

The respondents' opting for this coping strategy is mostly influenced by the level of social frustration and neutral and ambivalent anthropomorphic metaphors. This means that for the respondents choosing the "planning" coping strategy, the statuses of their communication partners in difficult situations can be important and so can their belonging to certain social groups. It has also been found at the trend level (the significance level being 0.019) that in the regression model, $23.4 \%$ of changes of the values of coping strategy "escapeavoidance" are due to the influence of the extent of social frustration and metaphors of difficult situations (see Table 4).

Table 4. Results of regression analysis of the impact of social frustration and metaphors of difficult situations on coping strategy "escape-avoidance".

\begin{tabular}{|c|c|c|c|c|}
\hline $\mathbf{R}$ & R Square & Adjusted R Square & F & Sig. \\
\hline 0.234 & 0.55 & 0.45 & 5.694 & 0.019 \\
\hline
\end{tabular}

The respondents' selecting this coping strategy is mostly influenced by the social frustration extent level. It has also been found at the trend level (the significance level amounting to 0.027 ) that in the regression model, $22.1 \%$ of changes of the values of the "distancing" coping strategy are due to the influence of the extent of social frustration and metaphors of difficult situations (see Table 5).

Table 5. Results of regression analysis of the impact of social frustration and metaphors of difficult situations on coping strategy "distancing".

\begin{tabular}{|c|c|c|c|c|}
\hline R & R Square & Adjusted R Square & F & Sig. \\
\hline 0.221 & 0.049 & 0.039 & 5.053 & 0.027 \\
\hline
\end{tabular}

The respondents' choosing this coping strategy is mostly influenced by neutral and ambivalent anthropomorphic metaphors. This means that for the respondents choosing the "distancing" coping strategy, the statuses of their communication partners in difficult situations can be important and so can their belonging to certain social groups. It has been found at the trend level (the significance level being 0.011) that in the regression model, $29.8 \%$ of changes of the values of coping strategy "Seeking social support" are due to the influence of the extent of social frustration and metaphors of difficult situations (see Table 6).

Table 6. Results of regression analysis of the impact of social frustration and metaphors of difficult situations on coping strategy "Seeking social support"

\begin{tabular}{|c|c|c|c|c|}
\hline R & R Square & Adjusted R Square & F & Sig. \\
\hline 0.298 & 0.089 & 0.070 & 4.71 & 0.011 \\
\hline
\end{tabular}

The respondents' choice of this coping strategy is mostly influenced by positive anthropomorphic and naturomorphic metaphors. This means that for the respondents who opt for coping strategy "Seeking social support" in difficult situations, the relationships 
with "their" people - relatives, close ones, friends, colleagues - are very important. They believe these people have positive social and psychological qualities and compare them to "positive" phenomena of nature. It has been found at the trend level (the significance level making 0.025 ) that in the regression model, $22.4 \%$ of changes of the values of the "undertaking the responsibility" coping strategy are due to the influence of the extent of social frustration and metaphors of difficult situations (see Table 7).

Table 7. Results of regression analysis of the impact of social frustration and metaphors of difficult situations on coping strategy "undertaking the responsibility".

\begin{tabular}{|c|c|c|c|c|}
\hline R & R Square & Adjusted R Square & F & Sig. \\
\hline 0.224 & 0.050 & 0.040 & 5.154 & 0.025 \\
\hline
\end{tabular}

The respondents' opting for this coping strategy is mostly influenced by negative anthropomorphic metaphors. This can mean that in a difficult situation, the respondents who choose coping strategy "undertaking the responsibility" categorize the people around them into "friends" and "aliens" ascribing negative qualities to the "aliens" and representatives of negatively evaluated social groups. It has also been found at the trend level (the significance level being 0.006 ) that in the regression model, $31.5 \%$ of changes of the values of coping strategy "Exercising self-control" are due to the influence of the extent of social frustration and metaphors of difficult situations (see Table 8). The respondents' selecting this coping strategy is mostly influenced by the level of social frustration and negative artifact metaphors. This can mean the respondents opting for the "Exercising selfcontrol" coping strategy may tend to perceive difficult situations as controllable ones and may tend to distance away from, to "objectify" the negative aspects of difficult situations and subjects they perceive as "aliens" and "adversaries".

Table 8. Results of regression analysis of the impact of social frustration and metaphors of difficult situations on coping strategy "Exercising self-control".

\begin{tabular}{|c|c|c|c|c|}
\hline R & R Square & Adjusted R Square & F & Sig. \\
\hline 0.315 & 0.099 & 0.080 & 5.328 & 0.006 \\
\hline
\end{tabular}

\section{Discussion}

The data obtained allow making the following conclusions within this sampling. The social frustration extent level has a negative impact on the selection of coping strategies "Planning", "Exercising self-control", "Positive reappraisal", "Seeking social support", on how the estimations of a difficult situation are differentiated and on the perception of its complexity. The social frustration extent level has a positive impact on the choice of coping strategies "escape-avoidance".

Negative artifact metaphors, metaphors of distinction and positive precedent name metaphors (i.e. perceiving difficult situations as ones under control, the stereotypically positive evaluations of "us" and "friends" and negative ones of "them" and "foes" - they are compared to inanimate objects, there is some "distancing away" from them) are associated with the "confrontational coping" strategy selected. Anthropomorphic metaphors expressing the importance of difficult situation communication partners' statuses for the subject, their belonging to certain social groups are associated with the "distancing" coping strategy. Positive anthropomorphic and naturomorphic metaphors of "friends", i.e. attributing positive social and psychological qualities to them, are associated with opting for the "Seeking social support" coping strategy.

Negative anthropomorphic and artifact metaphors, i.e. attributing negative qualities to "aliens" and representatives of negatively evaluated social groups, "distancing away" from them are associated with coping strategy "undertaking the responsibility". Negative artifact 
metaphors (i.e. perceiving the difficult situations as ones under control, comparing the "aliens", "adversaries" to inanimate objects) are associated with choosing "Exercising selfcontrol" coping strategy.

\section{Conclusions}

According to the authors, metaphors of difficult situations and their participants ("friends" and "aliens") reflect the subjects' perceiving the situations in scales of "short distance-long distance", "rational value-emotional value", and the parameters of "undertaking the responsibility - placing the responsibility with others", "stereotypical or differentiated image of the situation". The data obtain confirm the suggested hypotheses.

Thus, one's social frustration and ideas about a difficult situation influence one's coping strategies. They differ in individuals having different social frustration extent levels and different metaphors of the situations. The results of the research conducted are illustrative of the Russian coping behavior concept and are of practical importance for psychological counseling of individuals.

The most productive for the correction of unproductive forms of behavior in the student environment, work with social frustration, the development of tolerance in relation to various social groups, will be games and exercises aimed at developing a creative, and most importantly joint solution of various problems. Such games should contain elements of modeling, construction, transformation. The problems unfold in dynamics, and allow students to live dozens of conditional years in a reality that is compressed in time and events [13]. During the game, each student himself makes mistakes and finds successful solutions, enriching his personal experience, which is not forgotten, because "it was with me", opens up new resources and hidden opportunities. Various types of imitation-roleplaying games, in our opinion, can be successfully used to develop productive, individual coping-strategies of behavior in a student environment.

\section{Patents}

Funding: This research received no external funding

Conflicts of Interest: The authors declare no conflict of interest

\section{References}

1. T. Kryukova, Kostroma, 61 (2010) https://elibrary.ru/item.asp?id=20108405

2. T. Kryukova, T. Gushchina, Bulletin of N. A. Nekrasov Kostroma State University. Series: Pedagogy. Psychology. Social work. Youth studies. Sociokinetics, 3 (25), 194198 (2012) https://elibrary.ru/item.asp?id=18943001

3. M. Saporovskaya, Bulletin of N. A. Nekrasov Kostroma State University, 5, 198-201 (2013) https://elibrary.ru/item.asp?id=20935985

4. J. De la Fuente, I. Mañas, C.Franco, International Journal of Environmental Research and Public Health, 15(10) (2018) doi.org/10.3390/ijerph 15102230

5. M. Nicolas, G. Martinent, M. Drapeau, Frontiers in Psychology, (DEC), (2017) doi.org/10.3389/fpsyg.2017.02222

6. C. Vizoso, C. Rodríguez, O. Arias-Gundín, Higher Education Research and Development, 7, 1515-1529 (2018) doi.org/10.1080/07294360.2018.1504006 
7. P. Zepp, D. Potter, C. Haselwood, Family and Consumer Sciences Research Journal, 1, 73-86 (2018) doi.org/10.1111/fcsr.12274

8. E. Doryeva, Bulletin of N. A. Nekrasov Kostroma State University, 5, 191-194 (2013) https://elibrary.ru/item.asp?id=20935983

9. O .Bazhukova, The influence of frustration on the behavior of mentally retarded adolescent learners. Proceedings of A. I. Herzen Russian State Pedagogical University, 39, 53-59 (2011) https://elibrary.ru/item.asp?id=15790932

10. A. Bubnov, Bulletin of Voronezh Institute of the State Fire-Fighting Service of the Ministry of Emergency Situations of Russia, 1 (2), 52-54 (2012) https://elibrary.ru/item.asp?id=20142887

11. N. Chernova, Juridical journal of Samara University, 4, 69-73 (2015) https://elibrary.ru/item.asp?id=25322520

12. A. Korochentseva, E. Krasnova, O. Moysova, E3S Web Conf, Innovative Technologies in Science and Education (ITSE-2020), 210, (2020) https://doi.org/10.1051/e3sconf/202021018087

13. I. Mansurova, E. Krasnova, A. Korochentseva, To the problem of hope/hopelessness and its intensity in non-verbal behavior within the people representing the terrorist threat (4th International Multidisciplinary Scientific Conference on Social Sciences and Arts SGEM 2017, 24 - 30 August 2017). DOI: $10.5593 /$ sgemsocial2017/32/S11.058

14. E. Bityutskaya, Bulletin of Moscow University, Series 14. Psychology, 2, 40-56 (2013) https://elibrary.ru/item.asp?id=19136062

15. V. Gagai, K. Grineva, Bulletin of Chelyabinsk State Pedagogical University, 2, 33-43 (2013) https://elibrary.ru/item.asp?id=19409209

16. N. Kalinina, Difficult life situations from the standpoint of the contemporary schoolchildren: content and overcoming. Proceedings of Saratov University, New series. Series: Acmeology of education, Developmental psychology, 3 (7), 245-250 (2013) https://elibrary.ru/item.asp?id=21029974

17. I. Kochkareva, Omsk scientific bulletin, 4 (111), 192-194 (2012) https://elibrary.ru/item.asp?id=17996288

18. M. Kubyshkina, Theory and practice of social development, 13, 52-56 (2014) https://elibrary.ru/item.asp?id=22020355

19. G. Martiyanova, Scientific dialogue, $4 \quad$ (16), $74-84 \quad$ (2013) https://elibrary.ru/item.asp?id=19055751

20. O. Matsukevich, Bulletin of Tambov State University, 3 (107), 177-181 (2012) https://elibrary.ru/item.asp?id=17586340 